\title{
Measures of mindfulness and anxiety in OM meditators and non-meditators: A cross-sectional study
}

$\pi$
0
0
0
0
0
$\frac{1}{4}$

Deepeshwar Singh, Ashok Vinchurkar Suhas, Kalkuni Visweswaraiah Naveen, Hongasandra Ramarao Nagendra

Division of Yoga and Life Sciences, Swami Vivekananda Yoga Anusandhana Samsthana (S-VYASA), SVYASA, Bengaluru, Karnataka, India

Address for the Correspondence:

Dr. Deepeshwar Singh, Swami Vivekananda Yoga Anusandhana Samsthana, \# 19 Eknath Bhavan, Gavipuram Circle, KG Nagar, Bengaluru, Karnataka - 560 018, India.

E-mail: deepeshwar.singh@ gmail.com

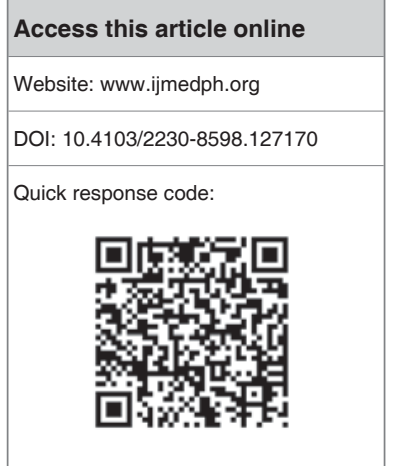

Background: Meditation has been shown to be an effective practice of mindfulness and psychological health. The aim of the study was to explore this relationship and to investigate the role of meditation on mindfulness skills and psychological health. Materials and Methods: Sixty-seven long-term ' $\mathrm{Om}^{\prime}$ ' meditation practitioners and equal number of normal healthy subjects matched to the meditators on age (meditators: $23.96 \pm 3.25$ years; non-meditators: $21.72 \pm 3.44$ years), years of education (meditators: $15.13 \pm 1.57$ years: non-meditators: $14.12 \pm 1.76$ years) participated in the study. Anxiety and mindfulness were measured by the State-Trait Anxiety Inventory (STAI) and Freiburg Mindfulness Inventory (FMI), respectively. Statistical analyses were carried out using the Statistical Package for Social Sciences (SPSS) software version 18.00 (SPSS Inc., Chicago, USA). The mindfulness and state and trait anxiety scores were analyzed using one-way analysis of variance (ANOVA) and independent $t$-test. Results: The meditator group showed significantly lower state $(P<0.001)$ and total anxiety $(P<0.001)$ as compared to the nonmeditation group. 'Om' meditation practice was positively correlated to mindfulness $(P<0.001)$, acceptance $(P<0.001)$, and presence $(P<0.05)$; and negatively correlated to state $(P<0.01)$ and total anxiety $(P<0.001)$. Conclusions: The practice of meditation was associated with higher levels of mindfulness and lower levels of psychological anxiety.

Key words: Anxiety, mindfulness, Om meditation, state and trait

\section{INTRODUCTION}

Mindfulness meditation is an ancient concept, grounded in a wide range of spiritual and religious traditions, including Yoga, Tai Chi, Buddhism, Zen, Taoism, Hinduism, etc. In Buddhist literature, mindfulness is described as the awareness that emerges through paying attention, on purpose, in the present moment, and non-judgmentally to the unfolding of experience moment by moment. ${ }^{[1]}$ Meditation can be defined as the intentional self-regulation of attention from moment to moment through which mindfulness is cultivated. ${ }^{[2]}$ In the Indian yogic tradition 'Om' is one of the fundamental symbol of meditation. It is the symbol of reality from which arises the three letters; namely, A, U, and $\mathrm{M} .{ }^{[3]}$ ' Om' meditation helps to enhance the awareness or mindfulness to all incoming sensations, emotions, and thoughts from moment to moment without focusing on any of them. ${ }^{[4]}$

During the last decade, scientific interest in meditation and mindfulness practice has an explosive and unprecedented surge. Long-term practice of meditation has been found to improve sustained attention, general well-being, mental health, enhance potency of positive feelings, and reduce anxiety. ${ }^{[5,6]}$ Additionally, meditation training has been shown to improve levels of mindfulness attention, working memory, and creativity. ${ }^{[7]}$ Practicing meditation may reduce psychophysiological arousal, improve concentration, selective attention, and visual scanning abilities compared to resting in a supine posture. ${ }^{[8,9]}$ Mental chanting of Om (with experience of 5-20 years) showed an increase in the efficiency with which sensory information was processed as revealed by activated higher neural centers, that is, the association cortices leading to a single thought state, and a subjective feeling of deep relaxation. ${ }^{[10]}$ A cyclical combination of yoga postures and supine rest in cyclic meditation (CM) improved memory scores immediately after the practice and decreased state anxiety more than rest in a classical yoga 
relaxation posture (shavasana) ${ }^{[9]}$ Mindfulness meditation and gentle yoga improve mood and affective processes and are associated with improvements in immune system functioning, stress, and emotional regulation. ${ }^{[11]}$ Meditation practice stabilizes the mind and decrease mental proliferation which are helpful to cultivate the ethical qualities, that is, compassion, mindfulness, loving kindness, and forgiveness. Practicing open monitoring meditation techniques try to enlarge the attentional focus to all incoming sensations, emotions, and thoughts from moment to moment without focusing on any of them is associated with increased theta activity. ${ }^{[12]} \mathrm{A}$ recent study found that 3 days of meditation training was effective at reducing pain ratings and sensitivity, as well as anxiety scores when compared to baseline and other manipulations, such as relaxation and a math distracter task. A similar training regimen improved mood and reduced heart rate when compared to a sham meditation and control group. ${ }^{[13]}$ Finally, meditation is defined as a family of complex emotional and attentional regulatory strategies developed for various ends, including the cultivation of well-being and emotional balance.

There are no previous studies reporting mindfulness levels of individuals practicing meditation on the syllable 'Om'. Therefore, the present study was designed to measure the levels of mindfulness and correlate the same to state and trait anxiety scores.

\section{MATERIALS AND METHODS}

\section{Subjects}

Sixty-seven Om meditators whose age ranged from 19 to 27 (23.96 \pm 3.25 years) were recruited for the study. An equal number of normal healthy male participants $(n=67)$ matched for their age, gender, and years of education were selected. $G$ power (two-tailed) was used to calculate the sample size. With alpha set to 0.05 and power at 0.95 , to get an effect size of 0.8 , the calculated sample size was 42 in each group. ${ }^{[14,15]}$ The non-meditator group had no previous experience of any form of meditation. All participants were recruited from Swami Vivekananda Yoga Anusandhana Samsthana (S-VYASA)—a Yoga University, Bangalore, India. These participants were recruited by announcements in the University newsletter and flyers on the notice boards. The inclusion criteria were as follows: i) No any chronic illness, particularly psychiatric, or neurological disorders; ii) male volunteers alone; iii) all meditators had been practicing meditation on the Sanskrit syllable, 'Om' for 30-200 min each day, for 5 days in a week; and iv) had a minimum of 1 year experience in meditation. None of the potential participants were excluded using these conditions. The demographic indices of meditators and non-meditators are outlined in Table 1 . Signed informed consent was obtained from all participants following a detailed explanation of the study. The study was approved by the Institutional Ethics Committee of the S-VYASA University.

\section{Assessments}

This cross-sectional survey aimed to collect data concerning mindfulness and state and trait anxiety using the Freiburg

\begin{tabular}{lc}
\multicolumn{2}{l}{ Table 1: Mean group differences of two groups } \\
and standard deviations \\
\hline Characteristics & Mean \pm SD \\
\hline Mean age & \\
$\quad$ Meditators & $23.6 \pm 3.25$ \\
$\quad$ Non-meditators & $21.72 \pm 3.44$ \\
Years of education & \\
$\quad$ Meditators & $15.13 \pm 1.57$ \\
$\quad$ Non-meditators & $14.12 \pm 1.76$ \\
Meditation experience (years) & \\
$\quad$ Meditators & $7.85 \pm 2.37$ \\
\hline
\end{tabular}

Mindfulness Inventory (FMI) and State-Trait Anxiety Inventory (STAI), respectively.

The description of measurements are given below.

\section{The FMI}

We used one-dimensional 14-item short version of FMI which was found to be semantically robust and psychometrically stable (Cronbach's alpha $=0.83) .{ }^{[16]}$ All items were scored on a 4-point Likert scale (0: rarely; 1 : occasionally; 2 : fairly often; 3 : almost always). Scores range from 8 to 32, with higher scores indicating higher levels of mindfulness. The FMI measures trait mindfulness and has been shown to have good psychometric properties including a high internal consistency (alpha of 0.86 in an initial validation study), and it has been shown to correlate positively with health indicators. ${ }^{[17]}$ Furthermore, the scale was able to differentiate between mindfulness practitioners and non-practitioners. The two proposed subfacets of the FMI, presence (items 1,2,3,5,7,10), and acceptance (items 4,6,8,9,11,12,14) was then tested separately. This scale is semantically independent from a Buddhist or meditation context and is applicable to all population groups.

\section{State and Trait Anxiety Scale}

The anxiety levels were assessed using a questionnaire 'State-Trait Anxiety Inventory' (STAI). ${ }^{[18]}$ This is a self-report assessment anxiety scale, which includes separate measures of state and trait anxiety. State anxiety (S-anxiety) is defined as a transitory emotional state characterized by consciously perceived feeling of tension and apprehension. Trait anxiety (T-Anxiety) refers to relatively stable individual differences in anxiety proneness. Depending on the characteristics of the stressful stimulus conditions, individuals experience differential levels of state anxiety as a function of their level of trait anxiety. The STAI consists of two separate subscales that contain 20 items each. The items are in the form of statements people used to describe themselves. The essential qualities evaluated are feelings of apprehension, tension, nervousness, and worry. Both subscales (S-Anxiety and T-Anxiety) use a 4-point Likert scale to allow the subject to show how often or how much each question applies to them in both situations. It has high internal consistency with Cronbach's alpha of 0.73 . Also, the test is designed to take only $20 \mathrm{~min}$ at the maximum to reduce the amount of fluctuations in S-Anxiety that could become apparent if the test was to go for a long period of time. 
These questionnaires were showing the relations among mindfulness and anxiety in participants of meditator and non-meditator groups.

\section{Data collection}

Each participant was assessed in 2 consecutive days at the same time. Participants were requested to use any necessary visual aids (i.e., glasses and contact lenses). On the day 1, participants in each group carried out the FMI first followed by the STAI on the day 2 at a time. To ensure each item was carefully considered and participants were advised they had an unlimited amount of time to complete the questionnaire. Participants received a recording blank with the front page on top and a pencil without an eraser. Participants were instructed as per the instructions stipulated on the manual of the questionnaires. Testing began once participants had confirmed they understood the given instructions. Participants were advised to provide answer as honestly and spontaneously as possible for every statement. The scoring was done by a person who was unaware when the assessment was made and whether the assessment was meditation group or control group.

\section{Statistical analysis}

The scores were analyzed using one-way analysis of variance (ANOVA). One-way ANOVA compared mindfulness and state and trait anxiety scores and independent $t$-test were used to compare mindfulness and anxiety of the data in 'Om' meditators and nonmeditators. Partial correlation ( $\mathrm{r}$ ) with meditation experience and anxiety and mindfulness is given in Table 1 . All statistical analyses were computed at $P \leq 0.05$, two-tailed, using PASW Statistics 18.00 (SPSS Inc., Chicago).

\section{RESULTS}

The age and years of education status were reported no significant difference in the meditation and non-meditation groups. An independent sample $t$-test and one-way ANOVA were performed to assess the difference in state and trait anxiety, and mindfulness in both the groups. The groups mean values $\pm \mathrm{SD}$, Cohen's d (effect size) for age, years of education, FMI mindfulness (mindfulness, acceptance, and presence), and STAI scores (state, trait, and total scores) are given in Table 2. The analysis on the FMI scores showed that participants in meditation group reported higher mindfulness scores in all three factors, mindfulness $(\mathrm{F}=3.85, P>0.001, t=$ 5.01), acceptance $(\mathrm{F}=7.152, P<0.001, t=4.78)$, and presence $(\mathrm{F}=1.85, P=0.038, t=2.10)$ compared to the participants in the non-meditation group. The non-meditation group shows higher anxiety as their state scores of STAI were higher than participants in the meditation group. Results showed that 'Om' meditation group have significantly higher mindfulness and less state anxiety compared to non-meditator group in the age-matched control group.

\section{Partial Correlation (r) with meditation experience and anxiety and mindfulness}

As shown in Table 2, the STAI scale and FMI strongly correlated with years of meditation experience. There was negative correlation found between meditation experience and STAI scores (state, trait, and total anxiety), while there was a strong positive correlation between years of meditation practice and FMI mindfulness scores (acceptance and presence). There was also a positive correlation between years of meditation with age and years of education.

\section{DISCUSSIONS}

In the present study, long-term meditators reported significantly lower state anxiety and total anxiety scores of STAI and higher level of total mindfulness scores, acceptance, and presence of FMI compared to the non-meditators. There was a strong, positive, partial correlation between experience of meditation with the total scores of mindfulness, acceptance, and presence; while there was a negative correlation with state and total anxiety. The acceptance component of the mindfulness scale is related to the nonjudgmental acceptance of the situation, while mindfulness presence is related to the experience of the moment and a cognitive reelection of all actions. ${ }^{[19]}$ Meditation aims to teach more accepting relationship of one's thought rather than emphasizing the creating of more positive or adaptive thoughts. Longer meditation experience reported more frequent meditation with higher mindfulness and lower psychological distress. However, meditation techniques effectively showed, reduce self-reported state and trait anxiety. ${ }^{[5]}$

Several studies of meditation to date have reported correlations between self-reported mindfulness and psychological health. For example, Lykins and Baer (2009) ${ }^{[20]}$ reported significantly higher

\begin{tabular}{|c|c|c|c|c|c|c|c|}
\hline Characteristic & & Meditators & Non-meditators & $F$ & $\begin{array}{l}\text { Percentage } \\
\text { change (\%) }\end{array}$ & $\begin{array}{l}\text { Cohen's d } \\
\text { (effect size) }\end{array}$ & $\begin{array}{l}\text { Partial correlation } \\
\text { (r) with meditation } \\
\text { experience }\end{array}$ \\
\hline \multirow{3}{*}{$\begin{array}{l}\text { State-Trait } \\
\text { Anxiety Inventory } \\
\text { (STAI) }\end{array}$} & S-STAI & $26.24 \pm 10.21$ & $32.75 \pm 8.29$ & $16.29^{* * *}$ & 24.81 & 0.700 & $-0.329^{* \star *}$ \\
\hline & T-STAI & $31.12 \pm 10.02$ & $33.44 \pm 7.36$ & 2.29 (NS) & 7.46 & 0.263 & -0.114 (NS) \\
\hline & Total-STAI & $57.36 \pm 9.87$ & $65.69 \pm 10.57$ & $22.01^{* * *}$ & 14.52 & 0.815 & $-0.0363^{\star * *}$ \\
\hline \multirow{3}{*}{$\begin{array}{l}\text { Freiburg } \\
\text { Mindfulness } \\
\text { Inventory (FMI) }\end{array}$} & Mindfulness & $45.42 \pm 5.22$ & $40.34 \pm 6.42$ & $25.05^{\star \star \star}$ & 11.18 & 0.868 & $0.355^{\star * *}$ \\
\hline & Acceptance & $24.53 \pm 4.21$ & $20.81 \pm 4.75$ & $22.86^{* * *}$ & 15.17 & 0.829 & $0.328^{* * *}$ \\
\hline & Presence & $20.89 \pm 3.49$ & $19.54 \pm 3.94$ & $4.42^{*}$ & 6.46 & 0.363 & $0.176^{*}$ \\
\hline
\end{tabular}

$* P<0.05, * * * P<0.001$ significance level, NS = Not significant 
levels of mindfulness, self-compassion, and overall sense of wellbeing; and significantly lower levels of psychological symptoms, rumination, thought suppression, fear of emotion, and difficulties with emotion regulation in meditators compared to non-meditators, and changes in these variables were linearly associated with extent of meditation practice. Linehan (1993) ${ }^{[21]}$ describes the development of mindfulness skills as a central goal of several behavior therapy, a leading mindfulness-based intervention. There was a strong consistency between extent of meditation practice with trait mindfulness as well as and other outcome variables, including fear of emotions, rumination, and behavioral correlations. ${ }^{[2]}$ Moreover, participants in the meditation group showed more mindfulness and were also more likely to cope with stress in adaptive ways, particularly using less avoidant-oriented strategies in stress situations. ${ }^{[23]}$ Mindfulness meditation has been found to regulate anxiety. In a recent study on mindfulness meditation reported significant reduction in state anxiety scores after meditation session. ${ }^{[24]}$ In the present study, we reported a strong positive correlation between experience of meditation and state anxiety and total anxiety; but there was no significant relation with trait anxiety. Our findings are consistent with previous studies that have found an inverse relation between mindfulness, stress, and state anxiety.

The trait anxiety represents a generalized tendency to be fearful, worried, and apprehensive about the future. It also reflects individual differences in the frequency and intensity with which anxiety states have been manifested in the past. The stronger trait anxiety may report more intense elevations in state anxiety in a threatening situation. There was no significant difference in trait anxiety of meditators and non-meditators in the present study because participants in both the groups were young and healthy. The immediate effect of a 30 min practice of a meditation technique called CM on state and trait anxiety was measured in normal healthy volunteers, which showed significantly better reduction in state anxiety after the $\mathrm{CM}$ and improve memory. ${ }^{[9]}$

Feldman et al., (2010) ${ }^{[25]}$ compared the immediate effects of mindful breathing to alternative stress management techniques (progressive muscle relaxation and loving-kindness meditation) in novice meditators, demonstrated greater decentering when compared to those receiving the two alternative interventions; there was also reduced frequency of repetitive thoughts and negative reactions to thoughts. These findings provide further evidence that cognitive aspects of meditation (e.g., mindful breathing) may create changes in cognitive processes ${ }^{[26]}$ associated with depression and anxiety (e.g., rumination) that are distinct from other validated stress management approaches. Mindfulness meditation is also documented to contribute for better coping in individuals in high stress work environments, such as medical students ${ }^{[5]}$ or business executives, and community members enrolled in a wellness program.

Although studies on neurophysiological changes reported the positive impact of meditation training on brain regions responsible for constructs that are often dysregulated in individuals with depression and anxiety disorders. Recently, majority of functional neuroimaging studies investigated brain regions like the anterior cingulate cortex (ACC) and the insula were shown to be involved in the development and maintenance of anxiety disorders. ${ }^{[27]}$ Meditation-related anxiety relief was associated with activation of the ACC, ventromedial prefrontal cortex, and anterior insula. Meditation-related activation in these regions exhibited a strong relationship to anxiety relief. During meditation, those who exhibited greater default-related activity (i.e., posterior cingulate cortex (PCC)) reported greater anxiety, possibly reflecting an inability to control self-referential thoughts. Meditation showed changes in activation of prefrontal cortex (PFC) and the Anterior Cingulate Cortex (ACC), as well as significant increases in alpha and theta activity. ${ }^{[28]}$ In addition, theta activity was found to be more common in experienced meditators, suggesting that greater meditation expertise may result in improved ability to self-regulate a state of deep relaxation. ${ }^{[2]}$ These findings are important to demonstrate a neurobiological impact of meditation on brain structures and regions (i.e., PFC, hippocampus, and limbic system) that are well-known to be affected in individuals with anxiety and depression. Several other studies show that meditation can reverse some abnormalities, like depression, anxiety, attention deficit, and posttraumatic stress disorder; producing salutary functional and structural changes in the brain. The mindfulness programs reported positive impact on symptoms of anxiety and depression, ${ }^{[30]}$ as well as improvements in sleep patterns and sustained attention. ${ }^{[31]}$ After several researches on meditation and mindfulness; however, clear mechanisms of change have yet to be identified. There are different behavioral, psychological, and biological pathways which have suggested how enhanced mindfulness may displace stress and anxiety-related illness and enhancing adaptive coping processes.

In summary, the present study suggested that, the intense practice of meditation on the symbol 'Om' may enhance mindfulness and reduce anxiety. Meditation techniques have been used to regulate the mind, emotions, and the responses in adverse psychological conditions. Therefore, meditation would be a mind body medicine which helps in the modulation of expectations, inner engagement, anxiety, and self-awareness.

\section{CONCLUSION}

In conclusion, the results suggest that mindful person may be less prone to anxiety-related problem. Mindfulness practice will help to increase awareness and problem solving strategies of the present moment which would facilitate effective processing as a means to enhance mental health and well-being. Further study of meditation and mindfulness may help to better disclose how the quality and depth of meditation influence mindfulness and enhancing adaptive strategies for anxiety and its related problems. Also, additional research is needed to clarify the mechanisms of change that are responsible for the beneficial effects of meditation on both psychological and physical health. 


\section{ACKNOWLEDGMENTS}

The present study was supported by Swami Vivekananda Yoga Research Foundation (S-VYASA) is gratefully acknowledged.

\section{REFERENCES}

1. Kabat-zinn J. Mindfulness-based interventions in context : Past, present, and future. Clin Psychol (New York) 2003;10:144-56.

2. Corsini RJ. Handbook of innovative therapy. Volume 204 of Wiley Series on Personality Processes. Ed. 2, illustrated, the University of Michigan: Wiley; 2001.

3. Sivananda S. Japa yoga: A comprehensive treatise on mantra-sastra. Shivanandanagar India: Divine Life Society; 1972.

4. Lutz A, Slagter HA, Rawlings NB, Francis AD, Greischar LL, Davidson RJ. Mental training enhances attentional stability: Neural and behavioral evidence. J Neurosci 2009;29:13418-27.

5. Shapiro SL, Schwartz GE, Bonner G. Effects of mindfulness-based stress reduction on medical and premedical students. J Behav Med 1998;21:581-99.

6. Wachholtz $\mathrm{AB}$, Pargament $\mathrm{KI}$. Is spirituality a critical ingredient of meditation? Comparing the effects of spiritual meditation, secular meditation, and relaxation on spiritual, psychological, cardiac, and pain outcomes. J Behav Med 2005;28:369-84.

7. Orme-Johnson D, Granieri B. The effects of the age of enlightenment governor training courses on field independence, creativity, intelligence, and behavioral flexibility. Scientific research on the Transcendental Meditation Program: Collected papers. Vol. 1. [Bad Bellingen, Germany]: Maharishi European Research University Press, 1977.

8. Sarang SP, Telles S. Immediate effect of two yoga-based relaxation techniques on performance in a letter-cancellation task. Percept Mot Skills 2007;105:379-85.

9. Subramanya $\mathrm{P}$, Telles $\mathrm{S}$. Effect of two yoga-based relaxation techniques on memory scores and state anxiety. Biopsychosoc Med 2009;3:8.

10. Telles S, Desiraju T. Recording of auditory middle latency evoked potentials during the practice of meditation with the syllable "OM". Indian J Med Res 1993;98:237-9.

11. Davidson RJ, Kabat-Zinn J, Schumacher J, Rosenkranz M, Muller D, Santorelli SF, et al. Alterations in brain and immune function produced by mindfulness meditation. Psychosom Med 2003;65:564-70.

12. $\mathrm{Mu} \mathrm{Y,} \mathrm{Han} \mathrm{S}$. Neural oscillations involved in self-referential processing. Neuroimage 2010;53:757-68.

13. Zeidan F, Johnson SK, Gordon NS, Goolkasian P. Effects of brief and sham mindfulness meditation on mood and cardiovascular variables. J Altern Complement Med 2010;16:867-73.

14. van den Hurk PA, Wingens T, Giommi F, Barendregt HP, Speckens AE, van Schie HT. On the relationship between the practice of mindfulness meditation and personality-an Exploratory Analysis of the Mediating Role of Mindfulness Skills. Mindfulness (N Y) 2011;2:194-200.

15. Erdfelder E, Faul F, Buchner A. GPOWER: A general power analysis program. Behav Res Methods Instrum Comput 1996;28:1-11.

16. Baer RA, Smith GT, Lykins E, Button D, Krietemeyer J, Sauer S, et al. Construct validity of the five facet mindfulness questionnaire in meditating and nonmeditating samples. Assessment 2008;15:329-42.

17. Leigh J, Bowen S, Marlatt GA. Spirituality, mindfulness and substance abuse. Addict Behav 2005;30:1335-41.

18. Spielberger CD, Gorsuch RL, Lushene R, Vagg PR, Jacobs GA. Manual for the state-trait anxiety inventory. PaloAlto: Consulting Psychologists Press; 1983.

19. Kohls N, Sauer S, Walach H. Facets of mindfulness - Results of an online study investigating the Freiburg Mindfulness Inventory. Pers Individ Dif 2009;46:224-30.

20. Lykins EL, Baer RA. Psychological functioning in a sample of long-term practitioners of mindfulness meditation. J Cogn Psychother 2009;23:226-41.

21. Linehan M. Skills Training Manual for Treating Borderline Personality Disorder. Diagnosis and Treatment of Mental Disorders. Illustrated, Guilford Publication; 1993.

22. Josefsson T, Larsman P, Broberg AG, Lundh LG. Self-reported mindfulness mediates the relation between meditation experience and psychological well-being. Mindfulness 2011;2:49-58.

23. Chang VY, Palesh O, Caldwell R, Glasgow N, Abramson M, Luskin F, et al. The effects of a mindfulness-based stress reduction program on stress, mindfulness self-efficacy, and positive states of mind. Stress Health 2004;20:141-7.

24. Zeidan F, Martucci KT, Kraft RA, McHaffie JG, Coghill RC. Neural correlates of mindfulness meditation-related anxiety relief. Soc Cogn Affect Neurosci 2013.

25. Feldman G, Greeson J, Senville J. Differential effects of mindful breathing, progressive muscle relaxation, and loving-kindness meditation on decentering and negative reactions to repetitive thoughts. Behav Res Ther 2010;48:1002-11.

26. Ramel1 W, Goldin PR, Carmona PE, McQuaid JR. The effects of mindfulness meditation on cognitive processes and affect in patients with past depression. Cognit Ther Res 2004;28:433-55.

27. Holzschneider K, Mulert C. Neuroimaging in anxiety disorders. Dialogues Clin Neurosci 2011;13:453-61.

28. Cahn BR, Polich J. Meditation states and traits: EEG, ERP, and neuroimaging studies. Psychol Bull 2006;132:180-211.

29. Chiesa A, Serretti A. Mindfulness-based interventions for chronic pain: A systematic review of the evidence. J Altern Complement Med 2011;17:83-93.

30. Baer RA. Mindfulness training as a clinical intervention: A conceptual and empirical review. Clin Psychol (New York) 2003;10:125-43.

31. Jha AP, Krompinger J, Baime MJ. Mindfulness training modifies subsystems of attention. Cogn Affect Behav Neurosci 2007;7:109-19.

How to cite this article: Singh D, Suhas AV, Naveen KV, Nagendra HR. Measures of mindfulness and anxiety in OM meditators and non-meditators: A cross-sectional study. Int J Med Public Health 2014;4:110-4

Source of Support: Nil, Conflict of Interest: None declared. 